\title{
LA COMUNICACIÓN INTERNA COMO HERRAMIENTA DE PREVENCIÓN Y GESTIÓN DEL CONFLICTO EN LAS ORGANIZACIONES HOTELERAS
}

\section{INTERNAL COMMUNICATION AS A TOOL FOR CONFLICT PREVENTION AND MANAGEMENT IN HOTEL ORGANIZATIONS}

María del Carmen Paradinas Márquez. ESIC Business \& Marketing School. España.

carmen.paradinas@esic,edu

\section{RESUMEN}

Este artículo difunde el estado del arte en términos de manejo de conflictos en las organizaciones en general y de cómo la comunicación interna dentro de éstas resulta una herramienta clave para ello, para luego enfocar la investigación en la revisión de la literatura sobre el manejo de conflictos laborales en las organizaciones hoteleras y su relevancia para generar ventajas competitivas ya que en la actualidad resulta relevante el uso de las nuevas tecnologías por parte de los clientes para contrastar opiniones de otros usuarios a cerca del hotel en el que van a elegir alojarse. Si el conflicto aflora entre el personal laboral de la organización, el cliente lo percibirá, su opinión será negativa en las redes y afectará finalmente a la cuenta de resultados. Para ello, se analizaron libros, publicaciones de divulgación e investigación científica, tesis doctorales y sitios contrastados para asegurar su fiabilidad, introduciendo varias ecuaciones de búsqueda selectiva que permitieron seleccionar los documentos más relevantes para la investigación. Los resultados indican que, aunque existen numerosos estudios sobre la gestión de conflictos en las organizaciones y otros muchos estudios sobre comunicación interna, muy pocos se centran en el análisis de la comunicación interna como uno de los elementos que contribuyen a la prevención y gestión del conflicto y aún menos en el sector turístico, detectando así la necesidad de profundizar en este importante motor de la economía.

PALABRAS CLAVE: comunicación interna; prevención del conflicto; gestión del conflicto; ámbito laboral; sector turístico; organización hotelera; ventaja competitiva.

\section{ABSTRACT}

This article presents the state of the art in terms of conflict management in organizations in general and how internal communication within them is a key tool for this, and then focus the research on reviewing the literature on labor conflict management in hotel organizations and its relevance to generate competitive advantages since it is currently relevant the use of new technologies by customers to contrast opinions of other users about the hotel in which they will choose to stay. If the conflict arises between the staff of the organization, the client will perceive it, their opinion will be negative in the networks and will ultimately affect the bottom line. To this end, books, publications and scientific research, doctoral theses and contrasted sites were analyzed to ensure their reliability, introducing several selective search equations that allowed the selection of the most relevant documents for research. 
The results indicate that, although there are numerous studies on conflict management in organizations and many other studies on internal communication, very few focus on the analysis of internal communication as one of the elements that contribute to the prevention and management of conflict and even less in the tourism sector, thus detecting the need to delve deeper into this important engine of the economy.

KEYWORDS: internal communication; conflict prevention; conflict managemen; working environment; tourism sector; hotel organisation; competitive advantage.

\section{Cómo citar el artículo:}

Paradinas Márquez, M. (2019). La comunicación interna como herramienta de prevención y gestión del conflicto en las organizaciones hoteleras. Revista de Ciencias de la Comunicación e Información, 24(1), 41-57.

doi: http://doi.org/10.35742/rcci.2019.24(1).41-57

\section{INTRODUCCIÓN}

Este trabajo trata de afrontar la visión, análisis y gestión del conflicto en las organizaciones, y en particular, en las empresas turísticas hoteleras, mediante la revisión del estado actual de tema, estudiando el impacto del conflicto laboral de cara al cliente, qué sistemas de resolución de conflictos adopta la empresa, qué canales emplea para su gestión y cómo la comunicación interna resulta una herramienta a veces olvidada o dejada en un segundo plano frente a la comunicación externa, con las implicaciones emocionales, sociales y económicas que ello conlleva, ya que el personal laboral es el principal grupo de interés de la organización siendo el capital humano su principal recurso.

Los esfuerzos de investigadores y estudiosos se decantan, en su mayoría, por la comunicación externa, siempre más clara y visible en las organizaciones; pero resulta conveniente analizar y reflexionar en torno a un concepto que puede ser estimado como el germen de la revolución pendiente en el ámbito empresarial (Caldevilla, 2012).

Se realizará en primer lugar un estudio en cuanto al conflicto se refiere para más adelante profundizar en el conflicto en el ámbito organizacional. En segundo lugar se abordará el estado del arte en cuanto a la comunicación interna se refiere para luego poder comprender la importancia de la misma en la prevención y gestión del conflicto en las organizaciones turísticas hoteleras.

Es reiterada la afirmación de que el conflicto es inherente e inevitable a las relaciones humanas en general, que toda relación tiene potencial suficiente para generar conflicto ( Ramos et al., 2013; Gómez, 2013), pero dependiendo del entorno o el marco en el que se produzca, se hace necesario analizar el origen del mismo, las partes implicadas, el sector y otra serie de factores para poder afrontarlo de la manera más adecuada para su resolución dentro de las múltiples técnicas existentes al efecto (Gómez, 2013; Constantino y Sickles, 1997). Así, y al estar las organizaciones compuestas por personas, para Fried (2011), los conflictos y las crisis son endémicos a éstas, siendo lo importante el cómo se trabajan en los niveles 
de gestión de personal y organizacional, pudiendo ser algunos de esos conflictos agudos y otros crónicos.

La literatura confirma que la propia dinámica del trabajo en grupo provoca que haya interacción entre las personas en el ámbito laboral y ello genera relaciones conflictivas (Janssen, 2004).

Pero el análisis realizado no sólo se ha centrado en la resolución del conflicto sino en su gestión, dado que no todo conflicto es susceptible de resolverse y mucho menos de manera favorable para todas las partes implicadas. ¿Qué ocurre entonces en esas situaciones? En ocasiones surgen conflictos dentro de la organización, ya sean intergrupales o intragrupales (Benítez, Medina y Munduate, 2012) que, si bien no se solucionan, pueden ser gestionados de manera que no afecten a la productividad del empleado, del equipo y en general de las partes implicadas y así no impacte directamente en los costes de la empresa.

El término gestión de conflictos es más amplio que el de resolución de conflictos ya que la resolución expresa la reconciliación de intereses, mientras que la gestión de conflictos incluye tanto la reconciliación, como dirigir los conflictos destructivos hacia resultados constructivos (Yirik, Yildirim y Çentinkaya, 2015).

Es por ello que el concepto "solución" de conflictos, que siempre se ha entendido como eliminación del conflicto (ya sea en una vertiente evitativa o de ganar - perder) crece para incorporar también a su ámbito la gestión del mismo, unida a la identificación de los intereses de las partes y aislándolas de sus posiciones (Fajardo, 2015).

\subsection{UNA APROXIMACIÓN AL CONCEPTO DE CONFLICTO}

Existen numerosas definiciones del conflicto y dependiendo de la acepción que se tenga del mismo, hará que su afrontamiento se realice de una u otra forma, y el modo de afrontar estas situaciones influye, no solo en los posibles beneficios de la empresa o las condiciones laborales, sino también en la calidad y salud de la propia estructura empresarial (Merino, 2008).

Tradicionalmente el conflicto se ha entendido como algo negativo, como guerra, enfrentamiento, controversia, lucha, combate (París, 2003). De esta manera, culturalmente, pensamos que si nos encontramos en una situación de conflicto con alguna persona o grupo de ellas, ésta dará lugar a un combate o pelea donde sólo puede haber vencedores y vencidos (Die, 2013).

Hocker y Wilmot (2014) definen el término como

una lucha que se expresa al menos entre dos partes independientes que perciben que los otros poseen metas, recursos escasos e interferencias incompatibles que les impiden a ellos alcanzar las suyas". Mientras que para Coser (1956) "el conflicto es una lucha sobre valores o reclamo de estatus, poder y recursos escasos en los cuales el objetivo de una de las partes en conflicto es no solamente obtener valores deseados sino también perjudicar o eliminar a sus rivales. 
En esta investigación se han recogido las definiciones más empleadas por voces expertas en el estudio del conflicto, existiendo otras muchas en el mismo sentido y observándose tal y como manifiesta González (2010), que es común a todas ellas que los implicados en el conflicto perciben que sus intereses están siendo afectados, o pudieran serlo (pues si no son conscientes de ello, el conflicto no existe); y que las metas de cada una de las partes son incompatibles, referido a la percepción que tiene el grupo respecto al logro de sus metas, que si son afectadas, desencadenarán el conflicto.

Derivada de esta acepción negativa del conflicto, existen numerosas definiciones todas ellas en el mismo sentido (pelea, angustia, batalla,...), pero si realmente el interés de las partes es resolver el conflicto, resultará más adecuado emplear el término conflicto desde una perspectiva positiva y entenderlo como una situación enriquecedora, y es desde este punto de vista, desde el que se plantea esta investigación. Conflicto como oportunidad de aprender, de crecer, de desarrollar nuevos paradigmas que impulsen a las partes hacia el crecimiento personal y social (Gómez, 2013).

\subsection{EL CONFLICTO EN LAS ORGANIZACIONES}

La aparición de situaciones de conflicto en el seno de la organización implica una serie de consecuencias a distintos niveles y que afectan tanto a las personas que la integran, como a la propia organización generando una serie de costes tanto emocionales como económicos.

Las discrepancias surgen de las relaciones que se entablan entre los equipos profesionales, bien sea entre iguales, bien sea entre personas con distintas responsabilidades dentro de la organización, y será necesario comprender el conflicto considerando los factores que alteran su normal funcionamiento, debiendo analizar sus causas o motivos antes de intentar abordarlo.

Autores, como por ejemplo Redorta (2004) optan por ampliar el elenco inicialmente propuesto por otros expertos, identificando quince tipos de conflicto, sustentado en la teoría de que los conflictos siguen patrones de comportamiento reconocibles e identificables, encontrando así conflictos de recursos escasos, de poder, de autoestima, de valores, de identidad, de expectativas, de inadaptación, de información, de intereses, de relaciones personales, de inhibición, de legitimación, normativos, atributivos y estructurales.

Para gestionar el conflicto de forma efectiva, positiva y eficaz, es necesario, además de conocer ante qué tipo de conflicto nos enfrentamos, detectar de qué forma lo afrontarán las partes intervinientes. Para ello encontramos la clasificación de Rahim (1985) que parte de dos variables que aparecerán con mayor o menor intensidad: preocupación por los demás y preocupación por sí mismo. Y partiendo de esas variables establece cinco formas de afrontar el conflicto: acomodativo (se centra en tratar de satisfacer las preocupaciones de la otra persona prestando poca atención o ninguna a las propias); colaborativo (trabaja con la otra parte de forma cooperativa para tratar así de encontrar una solución que integre y satisfaga las preocupaciones de todos); evitativo (muestra falta de interés y atención hacia las preocupaciones de 
cualquiera de las partes, mostrando apatía, negligencia o incluso retirándose); competitivo (trata de conseguir sus propios interese a expensas de los intereses y preocupaciones de los demás); por último, compromiso (refleja una actitud pragmática prefiriendo encontrar una solución mutuamente aceptable que satisface parcialmente los intereses de ambas partes).

Hoy día, el conflicto en las organizaciones se está convirtiendo en un aspecto de interés a la hora de estudiar su eficacia y eficiencia. Hasta no hace mucho tiempo, los conflictos en el ámbito laboral eran considerados meros incidentes desagradables (Carnegie, 2011). Ahora las empresas son conscientes de que las consecuencias son importantes y provocan costes económicos y emocionales por lo que se hace necesario implantar técnicas de prevención y gestión del conflicto con el fin de evitarlos y además generar así importantes ventajas competitivas.

La falta de atención al conflicto puede generar graves situaciones si se permite o no se controla la escalada del mismo, tales como constantes discusiones, bajas laborales o incluso agresiones o mobbing, ya que los conflictos surgidos entre el personal de una organización por causas personales, interpersonales o derivados de la estructura y cultura de la propia organización son fuente de grandes pérdidas económicas y humanas (Baixauli, 2017).

Ya se hizo referencia al hecho de que la gestión del conflicto y su prevención tiene que tener una visión holística en el ecosistema empresarial ya que el conflicto afectará a diversos campos que en sí mismos se encuentran interrelacionados. Si un conflicto en el trabajo puede derivar en una baja laboral, es evidente que esa baja tiene un coste tanto para la empresa, como para la Administración Pública. Igualmente si el conflicto no se gestiona correctamente, puede derivar en salidas de los empleados de la empresa, provocando una alto rotación con los costes que ello conlleva (nuevos procesos de selección, formación, etc.). Por último, es necesario atender a las horas que tanto las partes implicadas en el conflicto dedican al mismo como las que dedican quienes ocupan cargos superiores, gerentes y otros mandos en atenderlo lo cual redunda en una disminución de la productividad.

Por tanto, se puede hablar de una clasificación de costes (Die, 2013):

1. Costes directos: entre los que se encuentran la asignación de recursos a la gestión de los conflictos, entre los que están abogados, gastos judiciales, gastos en mantenimiento de sistemas disciplinarios. Asimismo, están dentro de este primer apartado el coste de la gestión propia de todo conflicto por el personal con responsabilidad que, como señala Die (2013), puede rondar entre el $30 \%$ y $50 \%$ del tiempo del personal directivo.

2. Costes derivados: Son aquéllos que se producen después de manifestarse el conflicto y la gestión del mismo, también llamados costes indirectos. En este punto se incluye la pérdida de productividad, la disminución de la motivación, la pérdida de cooperación, los costes emocionales, los costes de imagen y el abandono del personal, bien de la organización o bien por rotación entre servicios del mismo sistema.

De lo expuesto, se desprende la necesidad de plantearse la reducción de costes en este ámbito. Existen muchos ejemplos de ello, Singer (1994), hace referencia a la 
cultura norteamericana en relación al aumento constante de los litigios civiles, y arroja un dato que así lo confirma: la población de Estados Unidos equivale al $5 \%$ de la población mundial, teniendo el $35 \%$ de los abogados de todo el mundo.

Por su parte Vinyamata (2002), habla de la pérdida en miles de millones debido a la reducción de la productividad causada por la dedicación a los conflictos que les supone tanto a las partes implicadas directamente como a los directivos, gerentes o superiores, estando por ejemplo, la mayor parte de la empresas por encima del umbral del nivel de bajas por enfermedad que sería del $4 \%$. Estas bajas no tendrían su consecuencia exclusivamente en enfermedades, sino que responden a un subterfugio para obtener unos días libres ante la necesidad de evadirse de un estrés casi insoportable para los trabajadores.

A este absentismo, que consiste en la baja laboral de los trabajadores, habría que añadir lo que se denomina el absentismo presencial, que se da cuando los trabajadores, aun acudiendo a su puesto de trabajo y en su horario, deciden no trabajar, reduciendo su productividad. En este sentido resulta importante señalar lo que Bayón (2008), denomina absentismo virtual, que se produce cuando el trabajador se encuentra delante del ordenador pero sin trabajar, utilizando el correo electrónico o las redes sociales, dedicándose a cuestiones personales que nada tienen que ver con el desempeño de su puesto de trabajo.

En esta línea, Vinyamata (2005) aporta los datos de las investigaciones realizadas por el Centro de Gestión de Conflictos de la Universidad de Harvard, que muestran que el impacto que tienen los conflictos internos, suponen la reducción en un $20 \%$ de la productividad (Thomas 1992; Ursiny y Bolz 2007).

La rotación de personal y la pérdida de productividad han hecho que cada vez con mayor intensidad, las empresas se preocupen por el valor de sus activos humanos y actúen en consecuencia (Colom, Sarramona y Vázquez, 1994).

\subsection{EL CONFLICTO EN LAS ORGANIZACIONES TURÍSTICAS}

Dada la importancia del sector turístico en el crecimiento y desarrollo en España, resulta especialmente relevante el estudio de las relaciones que se establecen entre las personas que integran una organización turística, siendo el componente de la diversidad un factor clave para el desarrollo de programas y actuaciones encaminadas a la resolución y/o gestión de conflictos internos y en los casos en que no es bien administrada se puede presentar un alto grado de rotación, una difícil comunicación así como una mayor cantidad de conflictos interpersonales (Amorós, 2007). Estos conflictos afectan a la estructura de la propia organización y a la imagen que ésta proyecta de cara al cliente, cuando las relaciones personales y profesionales se encuentran en un estado de desgaste sin que se afronte la situación o se afronte de forma incorrecta. El sector turístico se caracteriza por la diversidad y la multiculturalidad, en muchas ocasiones dentro de la propia empresa, lo que da lugar a que la gestión del conflicto y los soportes de comunicación se adapten a esa diversidad dependiendo del entorno cultural o social en el que se produzca. 
En las últimas décadas, los factores intangibles de las organizaciones han ido cobrando especial importancia a la hora de posicionarse en el mercado (Grant, 1996). Por ello, la valoración de la experiencia que el cliente perciba de estos intangibles, tales como la marca, la lealtad de los consumidores, el conocimiento del personal sobre las actividades, la cultura corporativa, etc., resulta de gran interés.

En la actualidad la sociedad muestra una importante tendencia hacia el uso de las redes sociales en internet para verter sus valoraciones de ocio y en ocasiones se emplean para elegir, no ya tanto en base al precio, sino a las opiniones del resto de usuarios lo que provoca que las empresas turísticas tengan que adaptarse a los cambios con cierta celeridad, lo cual a veces, en cuanto a la gestión del conflicto se refiere, hace que su abordaje no se realice de la forma más deseable.

La alta competitividad de la industria turística y el estrecho contacto del cliente con el factor humano de las organizaciones, requieren cuidar todos estos aspectos intangibles, con especial énfasis sobre aquellos relativos al personal de contacto directo con el cliente. El ecosistema empresarial turístico es un marco ideal para el estudio de estas cuestiones, dado que existe una alta rotación, escasa cualificación y notable diversidad en los equipos y plantillas en general (Paradinas, Merino y García - Muiña, 2018).

Conde y Amaya (2007) hacen referencia a la parte intangible reconocida dentro de lo que denominan producto hotelero considerando que el mismo

está formado por el conjunto de bienes y servicios que se ofrecen en el mercado, para el confort material y espiritual, en forma individual o en una gama muy amplia de combinaciones resultantes de las necesidades y deseos del consumidor al que le llamamos turista.

Así, un producto como el hotelero se puede definir como un conjunto específico de atributos que aporta al comprador, no solo el servicio de base característico de la clase de producto, sino también un conjunto de servicios secundarios, que constituyen elementos distintivos, susceptibles de influir en las preferencias de los compradores (Lambin, 1995 en Conde y Amaya, 2007). Por tanto, se puede inferir que existe una estrecha e importante relación entre el clima de servicio y la percepción del cliente, ya que si ese clima no es bueno, será transmitido de forma ineludible a la relación entre empleado y usuario del servicio/consumidor del producto hotelero.

En los últimos años, la calidad del servicio se ha convertido en uno de los temas centrales de preocupación para la dirección de las empresas, llegando incluso a incorporar sus excelencias en las correspondientes campañas de Marketing, especialmente por su capacidad para generar ventaja competitiva (Carmeli, 2008; García, 2001).

Las empresas analizan la calidad desde el punto de vista interno y externo y es necesario no pasar por alto el hecho de que las dinámicas internas de la organización pueden repercutir en las actitudes y los comportamientos de los empleados, lo que a su vez influye en las percepciones y experiencias del cliente sobre el servicio recibido (Benítez, Medina y Munduate 2012). Por ello, resulta 
imprescindible saber cuáles son los factores y los procesos internos que podrían favorecer o inhibir los niveles de calidad percibida por el cliente (Schneider, Salvaggio, y Subirats, 2002 en Benítez et al. 2012).

Por lo tanto se aprecia una relación entre los valores intangibles como la calidad del servicio, las percepciones, las experiencias del cliente, y el negocio y su desarrollo y por ello son necesarias una serie de herramientas que permitan transformar esos valores intangibles en tangibles para poder cuantificarlos y así otorgarles un valor cualitativo que pueda aplicarse dar a conocer cómo afectan a los resultados de la empresa. De esta manera se pueden prever actuaciones y tendencias de los públicos interesados estableciendo líneas de actuación y comunicación de la organización (Rodríguez, González y Caldevilla 2017) sin dejar de lado el aspecto interno de ésta última.

En las empresas turísticas el trabajo en equipo es ineludible de manera que las relaciones de cooperación y cordialidad entre los diferentes grupos de trabajo implicados en la prestación de servicio son esenciales para la efectividad de la organización (Hodson, 2009). Pero este trabajo en equipo, que requiere cooperación y cordialidad, también es fuente de conflictos relacionales intragrupales que puede generarse incluso debido a problemas personales que nada tiene que ver con el trabajo tales como gustos, ideas, valores... De hecho hay estudios que revelan que la existencia de conflictos relacionales dentro de los equipos obstaculiza el desarrollo del trabajo en grupo (Gamero, González-Romá y Peiró, 2008).

Con frecuencia, las organizaciones carecen del conocimiento, estrategias y habilidades necesarias para manejar los conflictos de manera eficaz. Éstas deben desarrollar un sistema que les permita abordar el conflicto de manera positiva en sus distintas fases (detección temprana, escalada y resolución). El manejo eficaz del conflicto en las organizaciones comienza mucho antes de que el conflicto aparezca. La cuestión no es si el conflicto aparece, ya que siendo inherente a las relaciones humanas, el conflicto aparecerá, el objetivo es garantizar una estructura empresarial y un clima laboral que favorezcan que el conflicto se pueda afrontar y gestionar minimizando sus costes y maximizando sus beneficios. El planteamiento se basa en la prevención del conflicto cuando sea posible y su gestión constructiva, cuando la prevención no sea posible o deseable (Merino, 2008).

Debido a la rapidez con la que varían las tecnologías y las habilidades requeridas, la consideración de los empleados como factor estratégico y competitivo requiere de empresas que inviertan en su formación y desarrollo (Barreto y Azeglio, 2013).

La gestión del capital humano está viviendo importantes cambios radicales en su concepto y aplicación y se está convirtiendo en una de las estrategias empresariales básicas esenciales para lograr las ventajas competitivas que tanto busca la empresa (Barreto y Azeglio, 2013). Igualmente estos autores manifiestan que para toda empresa moderna que se compromete con el desarrollo o gestión del capital humano, se considera que éste es el factor principal de la productividad y la fuente del progreso económico, debiéndose la competitividad de las empresas en gran medida a la calidad de sus recursos humanos. Por lo tanto, si como hemos visto, la gestión del conflicto dentro de la organización turística no se gestiona o se hace de 
forma incorrecta impactará directamente no solo en el capital humano sino que, aumentará los costes, reducirá la productividad, provocará altos niveles de rotación y/o absentismo, lo que restará ventaja competitiva.

Las sociedades contemporáneas necesitan constantemente la búsqueda de nuevas soluciones para todos los nuevos problemas; nuevas técnicas y nuevas estrategias deben estar siempre como objetivo de la organización y por ello los nuevos métodos para lograr nuevos resultados parecen ser la mejor opción para avanzar (Duque, 2017).

\subsection{LA COMUNICACIÓN INTERNA}

"El concepto de comunicación dentro de las organizaciones se basa en el modelo emisor, receptor, canal y mensaje" (Caldevilla, 2009).

Atendiendo la definición ofrecida por Kreps, que entiende que la comunicación interna es "el manejo de mensajes compartidos entre los miembros de la organización; resultado de la interacción humana que ocurre dentro de las organizaciones y entre los miembros de las mismas" (1990, p. 22), se podría ampliar el concepto con las aportaciones de La Porte (2001) en cuanto a que la referida comunicación permite la creación de relaciones eficientes entre los miembros de la organización de forma que mejoran la calidad del trabajo y la realización del mismo.

En cuanto al objeto de estudio se refiere, es decir a la relación entre la comunicación interna y la prevención y gestión de conflictos en la organización resulta relevante la apreciación incorporada al tema de estudio por Álvarez en cuanto a que "la comunicación interna es la base sobre la que pivotan todas las demás comunicaciones. O lo que es lo mismo, el éxito empresarial" (2007, p. 6). Y completando esta relación entre la comunicación interna y las buenas relaciones entre el personal de la organización la definición de Andrade resulta muy descriptiva:

Es el conjunto de actividades efectuadas por cualquier organización para la creación y mantenimiento de buenas relaciones con y entre sus miembros, a través del uso de diferentes medios de comunicación, que los mantengan informados, integrados y motivados para contribuir con su trabajo al logro de los objetivos organizacionales (1991, pp. 32-33).

En el ecosistema empresarial actual, el personal laboral cada vez demanda más una comunicación coherente, directa, fluida, abierta y bidireccional con sus superiores de forma que si se produce algún fallo en esa comunicación y el sistema falla, de nada sirve el correcto funcionamiento de las demás herramientas (tablón de anuncios, intranet...) si el contacto personal falla o se rompe (Álvarez, 2007) porque en muchas ocasiones dará lugar a posibles malentendidos, rumores o corrillos que en ocasiones serán un cultivo de conflictos internos en escalada que se podían haber evitado con una buena gestión de la comunicación interna, además de dar lugar a un descenso de la productividad, aumento de la incertidumbre y falta de credibilidad en la dirección. "La comunicación cara a cara, el encuentro, el diálogo jefe-colaborador ha sido, es y será -más allá de toda la parafernalia comunicacional que construyamos- el canal más efectivo para las comunicaciones internas" (Reyes, 2012). 
Es importante además resaltar la idea de que la simple difusión de informaciones no es comunicación (Filho, 2004 citado en Brönstrup, Godoy y Ribeiro, 2007), insistiendo que en lo que al ámbito de la gestión del conflicto se refiere, la misma debe ser bidireccional.

En toda organización se crean relaciones entre las personas que trabajan en ella, no solo relaciones ya establecidas por la propia organización en cuanto a la necesidad de trabajar juntos para lograr el plan establecido por la empresa para lograr los objetivos, sino que se van conformando otras más informales donde entran en juego los afectos y otra serie de comportamientos irracionales propios de la conducta humana (Moret y Arcila, 2011). Estas relaciones son espontáneas e informales, personales, sociales, no profesionales y ello puede dar lugar a un cierto descontrol de la comunicación o del mando por parte de la jerarquía (Miller y From, 1969, citado en Moret y Arcila 2011). Para evitar esas situaciones, el personal debe estar correcta y directamente informado por la gerencia de la identidad de la organización ya que "la desinformación o la ambigüedad ante diversos hechos importantes, puede ocasionar la aparición de rumores" (Caldevilla, 2009), mientras que si la comunicación interna se fundamenta en el conocimiento de las necesidades del público interno, se conseguirán crear lazos de fidelidad y confianza entre el personal que conforma la organización y la propia empresa.

El conflicto surge porque se dan una serie de variables que lo favorecen, entre ellas, como se ha referido, una comunicación defectuosa o inexistente o unos procesos de comunicación erróneos. Pero a su vez, la única forma de gestionarlos y/o de resolverlos es a través de la comunicación mediante procesos de negociación o mediación encaminados a dialogar, entenderse y avanzar para solventar el conflicto (Rojas y Arapé, 2001).

Según Pacheco (2005) el manejo de conflictos debería ser considerado otra de las competencias claves de la comunicación organizacional y convertir los conflictos en oportunidades de mejoramiento de dicha comunicación es una habilidad que deberían poseer todos los líderes de la organización. Para Parra de Párraga, Rojas y Arapé, uno de los factores que perturban a la empresa es la falta de comunicación, y una desacertada y mal dirigida política de comunicación, ya que si se da, se rompen las vías de acuerdo y entendimiento que resultan beneficiosos para la organización y ello "acarreará situaciones indeseables llegando incluso a afectar al clima organizacional, por lo cual los actores en manifiesto conflicto deben necesariamente establecer algún contacto que les permita indagar lo que verdaderamente desea el contrincante" (2008, p. 18).

\section{OBJETIVOS}

El objetivo de este estudio ha sido explorar la incidencia del conflicto en el seno de las organizaciones y en concreto de las organizaciones turísticas, cómo éste afecta a la calidad y al clima del servicio y cómo herramientas como la comunicación interna pueden ayudar fundamentalmente a prevenir la aparición del conflicto o a gestionarlo una vez que ya ha aparecido pero sin dejar que llegue a una fase de escalada tal, que resulte inabordable. 
Para ello se ha considerado el papel que juegan dentro de la gestión del conflicto los diferentes estilos de afrontamiento. Los resultados de la investigación realizada permite afirmar, por un lado, que el conflicto existe en todas las organizaciones, ya sea interpersonal o intragrupal; y por otro lado, que una mala o inexistente gestión del conflicto por parte de la organización afecta muy negativamente a la productividad y en concreto en las organizaciones turísticas, al clima del servicio lo cual es fácilmente percibido por el cliente.

Las empresas en la actualidad operan condicionadas por las nuevas tecnologías, por la ineludible rapidez de adaptación al cambio y por la necesidad de generar procesos innovadores que les permitan mantener al menos una cierta ventaja competitiva, pero en ocasiones se centran más en la innovación del producto que en la innovación de los procesos, lo que genera que en la mayoría de las ocasiones los procesos dentro de la organización no se adapten a situaciones que pueden llegar a generar importantes costes. Uno de esos procesos es la comunicación interna, también a veces dejada de la do por centrarse más en la externa, pero muy importante si se utiliza entre otras cosas como herramienta de preveción y gestión del conflicto.

Una correcta gestión del conflicto dentro del ámbito laboral de la organización no sólo ahorrará costes económicos sino emocionales ya que el hecho de que el capital humano sea menos productivo, no solo provoca pérdida de horas que el empleado no dedica a su trabajo sino que puede abocar a una baja laboral por estrés o depresión que afecta al propio sujeto, a la empresa e incluso a la Administración (De Dreu et al., 2004).

Este no será el único factor que haga que la organización turística gane ventaja competitiva, ya que además si evita una alta rotación, bajas, absentismo y un exceso de horas dedicadas a la gestión ineficaz del conflicto por parte del personal, los gerentes y directivos, ahorrará una importante cantidad de recursos que, destinados a otros procesos, harán que se posicione en el sector con mayor facilidad.

\section{METODOLOGÍA}

Este texto parte de una revisión bibliográfica de carácter sistemático de la materia objeto de estudio durante los años 2017 hasta agosto de 2019.

En primer lugar se ha partido de la definición del problema para, de esta manera, poder realizar una búsqueda bibliográfica acertada. Para ello se ha trabajado sobre el concepto de resolución de conflictos en primera instancia, para después reducirlo al ámbito de las organizaciones y posteriormente centrarse en las de carácter turístico, además de enfocar la otra vía de investigación en la comunicación interna en las organizaciones y analizar el estado del arte en cuanto a la relevancia de la misma en cuanto a la prevención y gestión del conflicto.

En esta revisión se han analizado libros, revistas de divulgación e investigación científica, libros de actas, tesis doctorales y sitios web contrastados para asegurar su fiabilidad, introduciendo diversas ecuaciones de búsqueda selectiva que han permitido seleccionar los documentos más relevantes para la investigación. 
Se han empleado criterios de búsqueda en inglés y en español en diversas bases de datos relacionadas con las ciencias jurídicas y humanidades, tales como: Organizational Conflict, Conflict Resolution, Conflict Mediation, Employee Conflict, Effective Conflict, Conflict Management, Tourism Conflict Management, Labor Conflict in Tourism Management o Conflicto Organizacional, Gestión de Conflictos, Resolución de Conflictos Laborales, Trabajo Emocional e el Sector Turístico y Conflicto Laboral en el Sector Turístico y Comunicación Interna.

La introducción de dichos criterios arrojó un resultado de más de quinientos artículos eligiéndose para su estudio únicamente los publicados a partir del año 2000, si bien en este artículo se citan autores cuyas publicaciones son anteriores. Ello es debido a que los mismos son reiteradamente citados por los autores cuya bibliografía se ha revisado y se ha considerado necesario incluirlos al tener gran peso en el ámbito de la gestión del conflicto y especialmente en el ámbito organizacional.

Una vez organizada de forma sistemática la bibliografía encontrada por relevancia, distinguiendo los principales documentos de los secundarios, se ha podido conseguir una estructura que ha permitido identificar las bases del tema de estudio.

Por último, se ha procedido al análisis de la información atendiendo a los documentos más útiles, bien por su contenido, bien por su estructura y contenido, si bien esta fase se ha realizado en paralelo a la primera por ser un proceso constante durante el desarrollo de la investigación.

Esta búsqueda bibliográfica ha hecho pensar que vale la pena continuar en la búsqueda y realizar hallazgos empíricos que puedan llevar a testar los cuestionamientos y las hipótesis que serán delineados, entre otras cuestiones, para poder por ejemplo, tal y como se ha mencionado, poder cuantificar el valor de los intangibles que tan relevantes son para la organizaciones y su relación con la cuenta de resultados y la creación de ventaja competitiva.

\section{RESULTADOS}

Si se quiere reducir el coste que produce la resolución de conflictos adversarial e implantar técnicas de prevención, gestión y resolución de conflictos, es necesario innovar como proceso imprescindible para la implantación de los conocidos como sistemas ADR (Alternative Dispute Resolutions), basados en la colaboración y voluntariedad y que se emplean para resolver conflictos fuera del ámbito judicial y resultan más ágiles, más económicas y emocionalmente menos costosas para las partes, pudiendo acudirse a ellas para resolver conflictos surgidos en el seno de cualquier ámbito en el que existan relaciones humanas (laboral, empresarial, familiar, vecinal, escolar...). Además resulta necesario innovar en cuanto a los procesos de comunicación interna haciéndolos ágiles, fluidos, bidireccionales y en los que el personal contratado se sienta escuchado e informado de todas las cuestiones que afecten a la empresa.

Es vital que las organizaciones turísticas sean conscientes de que la innovación no sólo es exclusiva de los productos tangibles o servicios y puede extenderse al propio proceso, a la organización o a la gestión del marketing (Barbosa y Dominique - 
Ferreira, 2012) y que para implantar nuevos procesos de gestión del conflicto incluso puede ser necesario cambiar la cultura empresarial o los sistemas de comunicación existentes hasta el momento. En la materia que nos ocupa, estaríamos hablando de innovación, de procesos, no de producto ya que el término innovación de procesos engloba la previsión de nuevas estrategias laborales, la actividad de proceso y la implantación del cambio en las complejas dimensiones humanas, tecnológicas y organizacionales de la empresa, buscando esta última, el desempeño. (Devenport, 1993 en Barbosa y Dominique - Ferreira, 2012). La innovación de procesos es típicamente aplicada en la organización mientras que la de producto o servicio está orientada al mercado (Freire, 2000).

La referida innovación pasa por introducir sistemas en la organización que permitan abordar el conflicto de la manera más adecuada, eficaz y eficiente posible.

La complejidad y diversidad de los asuntos a comunicar, más las diferentes características de los públicos, implica el desarrollo de distintas herramientas de comunicación, que además difieren dependiendo de la tipología de cada empresa y el número de empleados. Una pequeña o mediana empresa, cuyos trabajadores realizan sus tareas en un único centro productivo, no necesita los mismos soportes que una gran compañía con centros dispersos en distintas ubicaciones. (Álvarez, 2007).

Es fundamental estudiar primero el perfil del público para aplicar y adaptar los discursos organizacionales. El conflicto forma parte de la vida cotidiana, unos más complejos que otros, lo importante es saber enfrentársele y tener la suficiente agudeza cognitiva como para anticipar los posibles escenarios donde los contrincantes pueden actuar, para lograrlo hay que memorizar las reglas del juego y evitar su trasgresión por cualquier jugador (Adair, 1990; Bohórquez, 2000). El conflicto se instaura cuando existe desacuerdo, y al radicalizarse ambas partes negocian la utilización de mecanismos que viabilicen el posible arreglo compartido y beneficioso, para lograrlo se valen de la comunicación, como la salida más expedita. La comunicación es la mejor herramienta para la solución de conflictos, su empleo en el momento adecuado y de manera eficiente contribuye a despejar dudas, aclarar ideas y fijar posiciones, para lo cual los afectados deben respetar las diferencias respectivas y eso solo se consigue cuando hay comunicación.

\section{REFERENCIAS}

Álvarez, J. (2007). Comunicación interna. La estrategia del éxito. Razón y Palabra, (56), 1-6. Recuperado de http://www.razonypalabra.org.mx/anteriores/n56/jalvarez.html\#au

Amorós, E. (2007). Comportamiento Organizacional. En busca del desarrollo de ventajas competitivas. Lambayeque: Escuela de Economía USAT.

Andrade, H. (1991). Hacia una definición de la Comunicación Organizacional. La Comunicación en las Organizaciones. México: Trillas. 
Baixauli, E. (2017). La mediación empresarial. Una herramienta para la prevención del mobbing. Madrid: SEPIN.

Barbosa, B. M. y Dominique-Ferreira, S. (2012). La innovación de los procesos. Diferenciación en los Servicios turísticos. Estudios y Perspectivas en Turismo. (21), 963-976.

Barreto, A. y Azeglio, A. (2013). La problemática de la gestión del capital humano en las MiPymes de alojamiento turístico de la ciudad de Buenos Aires-Argentina. Estudios y Perspectivas en Turismo, (22), 1.14-1.159.

Bayón, F. (2009). Organizaciones y recursos humanos. Economía de la Empresa. Editorial Síntesis. Madrid.

Benítez, M., Medina, F. J. y Munduate, L. (2012). La gestión de conflictos relacionales en las organizaciones de servicios. Anales de Psicología, 28(1), 139- 49.

Brönstrup, C., Godoi. E y Ribeiro, A. (2007). Comunicación, lenguaje y comunicación organizacional. Signo y Pensamiento, XXVI(51). Recuperado de http://www.redalyc.org/articulo.oa?id=86005104

Caldevilla, D. (2009). Comunicar en situaciones de crisis. Vivat Academia, Revista de Comunicación, XII(105), 1-27.

Caldevilla, D. (2012). Claves de la comunicación interna como sistema de comunicación empresarial actual. Diálogos de la Comunicación. Revista académica de la Federación Latinoamericana de Facultades de Comunicación social. FELAFACS, (83), 1-19.

Carmeli, A. (2008). Top team behavioural integration and the performance of service organizations. Group \& Organization Management, (33), 712-735.

Carnegie, D. (2011). Las cinco habilidades esenciales para tratar con las personas. Barcelona: Ediciones Elipse.

Colom, A., Sarramona, J. \& Vázquez, G. (1994). Estrategias de formación en la empresa. Madrid: Ed. Nancea.

Conde, E. M. y Amaya, C. M. (2007). El producto hotelero: visto como un conjunto de atributos tangibles e intangibles. Gestión Turística, (8), 75-84.

Constantino, C. A. y Sickles Merchant, C. (1997). Diseño de sistemas para enfrentar conflictos. Barcelona: Granica D.L. 
Coser, L. (2004). The functions of social conflict. N.York (1956). En Domínguez Bilbao, R. y García Dauder, S. Introducción a la teoría del conflicto en las organizaciones. Madrid: Servicio de Publicaciones de Universidad Rey Juan Carlos.

De Dreu, C. K. W., Van Dierendonck, D. \& Dijskstra, M. T. M. (2004). Conflict at work and individual well-being. International Journal of Conflict Managemen, 15, 626.

Die, F. (2013). Análisis de las necesidades jurídicas y metodológicas para la implementación de sistemas ADR en centros hospitalarios públicos (Tesis Doctoral). Universidad Complutense de Madrid, Madrid.

Duque, E. (2017). The Human Capital as an Engine of Sustainable Development: Analysis of the National and Regional Reality of Portugal. Revista Portuguesa de Estudos Regionais, (46).

Fajardo, P. (2015). Cooperar como estrategia. Sobre el uso de métodos alternativos para la solución de los conflictos de las empresas. (Tesis Doctoral). Universidad Pontificia de Comillas, Madrid.

Freire, A. (2000). Inovação: novos produtos, serviços e neócios para Portugal. Lisboa: Ed. Verbo.

Fried, D. (2011). Afrontamiento generativo de crisis y conflictos en organizaciones. Revista Persona, (14), 11-40.

Gamero, N., González-Romá, V., \& Peiró, J. (2008). The influence of intra-team conflict on work teams' affective climate: A longitudinal study. Journal of Occupational and Organizational Psychology, 81, 47-69.

García, E. (2001). Calidad de servicio en hoteles de Sol y playa. Valencia: Editorial Síntesis.

Gómez, G. (2013). El conflicto en las Organizaciones y Mediación. Málaga: Universidad Internacional de Andalucía.

González, Y. (2010). El conflicto organizacional. Una solución constructiva. Pensando Psicología, 6(11), 15.

Grant, R. (1996). Toward a Knowledge-Based Theory of the Firm. Strategic Management Journal, 17(Winter Special Issue), 109-122.

Hocker, J. \& Wilmot, W. (2014). Interpersonal conflict. Mc Graw Hill Education.

Hodson, G. (2009). The puzzling person-situation schism in prejudice re-search. Journal of Research in Personality, 43, 247-248. 
Janssen, O. (2004). The barrier effect of conflict with superiors in the rela-tionship between employee empowerment and organizational commit-ment. Work \& Stress, 18, 1-10.

Kreps, G. L. (1990). La comunicación en las organizaciones. Wilmington: Addison Wesley Iberoamericana.

La Porte, J. M. (2001). Entusiasmar a la propia institución. Gestión y comunicación interna en las organizaciones sin ánimo de lucro. Madrid: Ediciones Internacionales Universitarias (Eiunsa).

Merino, C. (2008). Gestión estratégica de conflictos en ámbito empresarial: Transferencia desde la práctica de la mediación. I Forum Internacional de Mediaçao Empresarial. Sao Paulo.

Moret, J. y Arcila, C. (2011). Comunicación interna e informal en las organizaciones. Temas de Comunicación, 22, 7-23.

Pacheco, R. (2005). Competencias claves para la comunicación organizacional. Chasqui, Revista latinoamericana de Comunicación, 90, 70-75.

Paradinas, M. C., Merino, C. y García-Muiña, F. E. (2018). La gestión estratégica del conflicto laboral en el ámbito de las organizaciones turísticas. Libro de Actas Congreso CUICIID 2018.

Paris, S. (2003). La conflictología. Un aprendizaje positivo de conflictos". Reseña de Aprender del conflicto. Conflictología y Educación de Eduard Vinyamata. Convergencia. Revista de Ciencias Sociales, 10(33), 315-328.

Parra De Párraga, E., Rojas, L. R. y Arapé, E. (2008). Comunicación y Conflicto: El arte de la negociación. Negotium, 4(10), 17-35. Recuperado de www.revistanegotium.org.ve

Rahim, M. A. (1985). A Strategy for Managing Conflict in Complex Organizations. Human Relations, 38(1), 81-89.

Ramos, A. L., et al. (2013). Conflicto, destello de oportunidades. Revista Plumilla Educativa, (12), 250 - 268.

Redorta, J. (2004). Como analizar los conflictos: la tipología de los conflictos como herramienta de mediación. Barcelona: Paidós Ibérica.

Reyes, J. (2012). Las cuatro dimensiones de la comunicación interna. Centro de Estudios en Diseño y Comunicación, 40, 127-138.

Rodríguez, J., Gonzálvez, J. E., Caldevilla, D. (2017). Aplicación de un modelo y sistema de medición de variables intangibles en el entorno del negocio empresarial. Revista Latina de Comunicación Social, 72, 560-573. 
Rojas, L. R. y Arapé, E. (2001). Comunicación, conflicto y negociación. TELOS, 3(3), 367-373.

Singer, L. R. (1996). Resolución de conflictos: técnicas de actuación den los ámbitos empresarial, familiar y legal. Barcelona: Ediciones Paidós Ibérica, S.A.

Thomas, K. (1992). Conflict and negotiation processes in organizations. In M. D. Dunnette \& L. M. Hough (Eds.), Handbook of industrial and organizational psychology (pp. 651-717). Palo Alto, CA, US: Consulting Psychologists Press.

Ursiny, T. \& Bolz, D. (2007). The top performer's guide to conflict. Naprville: Soucebooks, Inc.

Vinyamata, E. (2002). Los conflictos explicados a mis hijos. Barcelona: Plaza \& Janés Editores S.A.

Vinyamata, E. (2005). Conflictología. Curso de resolución de conflictos. Barcelona: Ariel S.A.

Yirik, S., Yildirim, B. I. \& Çentinkaya, N. (2015). A study on conflict management and conflict resolution in hospitality organizations. International Journal of Arts \& Sciences, 8(8), 77-88. 